\title{
Investigation of Unmeasured Parameters Estimation for Distributed Control Systems
}

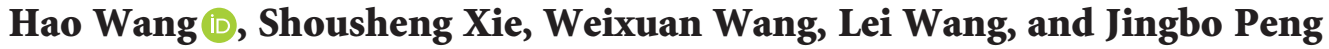 \\ Aeronautics Engineering College, Air Force Engineering University, Xi an 710038, China \\ Correspondence should be addressed to Hao Wang; 15129822645@163.com
}

Received 11 September 2019; Revised 10 February 2020; Accepted 20 February 2020; Published 29 March 2020

Guest Editor: Wonhee Kim

Copyright (c) 2020 Hao Wang et al. This is an open access article distributed under the Creative Commons Attribution License, which permits unrestricted use, distribution, and reproduction in any medium, provided the original work is properly cited.

\begin{abstract}
The problem of unmeasured parameters estimation for distributed control systems is studied in this paper. The Takagi-Sugeno fuzzy model which can appropriate any nonlinear systems is employed, and based on the model, an observer-based fuzzy $H \infty$ filter which has robustness against time-delay, external noise, and system uncertainties is designed. The sufficient condition for the existence of the desired filter is derived in terms of linear matrix inequalities (LMIs) solutions. Moreover, the underdetermined estimation problem in which the number of sensors available is typically less than the number of state variables to be estimated is specifically addressed. A systematic method is proposed to produce a model tuning parameter vector of appropriate dimension for the estimation of the filter, and the optimal transformation matrix is selected via iterative solution to minimize the estimated error. Finally, a simulation example for turbofan aeroengine is given to illustrate the effectiveness of the proposed method, and the estimated error is less than $2.5 \%$.
\end{abstract}

\section{Introduction}

Along with the continuous development of computer and network technology, distributed control systems (DCSs) have gradually become a new trend and attract much attention $[1,2]$. Compared with traditional point-to-point control systems, DCSs have advantages such as high reliability, reduced weight, low cost, and ease of maintenance $[3,4]$. The DCSs provide the low-level processing function via intelligent unit and are more conductive to the implementation of complex control algorithms $[5,6]$. However, the signal of DCSs is transmitted via bus network, which brings new challenges, such as networkedinduced time-delay, packet dropout, and packet disordering.

In practice, many parameters are difficult to obtain due to the limitation of the system itself (for example, the sensors cannot be installed in poor working conditions) or cost constraints. Thus, the estimation of unmeasured system parameters is significantly important, since the estimated parameters can not only be used in the design of controller but also for online monitoring $[7,8]$. For the estimation problem of unmeasured system parameters, there are two main methods: (1) high-precision mathematical model [9]; (2) filtering technique and its extension methods [10]. The first method is to establish the mathematical model based on the structure of system to solve the unmeasured parameters. However, the modeling process is often cumbersome and complicated. And for some systems, the structure is extremely complicated and cannot be accurately modeled. Moreover, since this method generally requires iterative calculation, the amount of calculation is large.

For the second method, the estimation of unmeasured parameters is based on the value of measurable parameters using the filtering technique. Because this method uses a recursive algorithm, it is easy to implement on computer and meet the requirements of accuracy and real-time. Among the filters, the $H_{\infty}$ filter stands out because it has robust stability against external noise without priori 
knowledge of noise and precise mathematical model [11-13].

However, in some cases, the number of sensors available is typically less than the number of state variables to be estimated, which is an underdetermined estimation problem and cannot be solved directly. A common approach to address this shortcoming is to estimate a subset of the unknown parameters and assuming that others remain unchanged [14-16]. Although this approach enables online filter-based estimation, it will introduce error in the accuracy of overall estimation application. If any of the parameters which are assumed unchanged moves away from their nominal values, the estimates can no longer represent the true parameters. Litt [17] presented a novel approach based on singular value decomposition (SVD) that selects a model tuning parameter vector of low enough dimension. The model tuning parameter vector is constructed as a linear combination of all unmeasured parameters and the transformation matrix is generated by selecting the $k$ most significant terms of singular values, where $k$ is the number of available sensors. Similarly, the SVD is also used in [18] to determine which parts of the systems are observable if the whole system is unobservable. However, the unaccounted terms of singular values may also have great importance on the accuracy of overall estimation application even if they are small under some circumstances.

Furthermore, in [19], the transformation matrix is selected to minimize the theoretical mean squared estimation error at a steady-state open-loop linear design point. But the estimation results are affected by the parameter perturbation, and there are many limitations in application. Moreover, all the approaches above do not consider the impact of time-delay and uncertainties of the system. Thus, it has great significance and practical value to propose a novel approach for the underdetermined estimation problem.

Besides, due to the strong nonlinear fitting characteristics of complex nonlinear systems, the Takagi-Sugeno (TS) fuzzy model has been widely used in the study of nonlinear systems since it was proposed [20-22]. Thus in this paper, an observer-based fuzzy $H \infty$ filter is constructed, and the unmeasured parameters estimation problem for T-S fuzzy distributed control systems with time-delay and parameters perturbation is studied.

The contributions of this paper can be concluded as follows: (i) a model tuning parameter vector of appropriate dimension is produced for the estimation of the filter; (ii) a systematic method is proposed for the selection of optimal transformation matrix to minimize the estimated error via iterative solution; (iii) the parameter perturbation is considered, so that the designed filter has a certain range of margins of disturbance.

The remainder of this paper is organized as follows. Section 2 introduces the modeling method of DCSs and the observer-based fuzzy $H \infty$ filter. The main results are presented in Section 3, including the analysis and synthesis of the filtering error system.Section 4 presents the approach of optimal transformation matrix selection. Numerical simulation results are shown in Section 5 and finally conclusion and a discussion of further application of the method are presented.

\section{Problem Formulation}

Consider the DCSs with time-delay, which can be described by a class of T-S fuzzy model as follows:

Plant rule $i$ : If $f_{1}(t)$ is $\Phi_{1}^{i}$ and $\cdots f_{g}(t)$ is $\Phi_{g}^{i}$, then

$$
\begin{aligned}
\dot{x}(t) & =\left(\mathbf{A}_{i}+\Delta \mathbf{A}_{i}\right) \mathbf{x}(t)+\left(\mathbf{B}_{i}+\Delta \mathbf{B}_{i}\right) \mathbf{u}(t-\tau(t))+\mathbf{L}_{i} \mathbf{h}(t)+\left(\mathbf{E}_{1 i}+\Delta \mathbf{E}_{1 i}\right) \mathbf{w}(t), \\
\mathbf{y}(t) & =\left(\mathbf{C}_{i}+\Delta \mathbf{C}_{i}\right) \mathbf{x}(t)+\left(\mathbf{D}_{i}+\Delta \mathbf{D}_{i}\right) \mathbf{u}(t-\tau(t))+\mathbf{M}_{i} \mathbf{h}(t)+\left(\mathbf{E}_{2 i}+\Delta \mathbf{E}_{2 i}\right) \mathbf{w}(t), \\
\mathbf{z}(t) & =\left(\mathbf{F}_{i}+\Delta \mathbf{F}_{i}\right) \mathbf{x}(t)+\left(\mathbf{G}_{i}+\Delta \mathbf{G}_{i}\right) \mathbf{u}(t-\tau(t))+\mathbf{N}_{i} \mathbf{h}(t) \\
\mathbf{x}(t) & =\phi(t), \\
t & \in\left[-\tau_{m}, 0\right],
\end{aligned}
$$

where $\Omega_{j}^{i}(i=1, \ldots, r ; j=1, \ldots, g)$ denotes the fuzzy set, $r$ denotes the number of IF-THEN rules, and $f_{j}(t)$ denotes the premise variable. $\mathbf{x}(t) \in \mathbb{R}^{n}$ is the vector of state variables; $\mathbf{u}(t) \in \mathbb{R}^{m}$ is the vector of control inputs; $\mathbf{y}(t) \in \mathbb{R}^{p}$ is the vector of measured outputs; $\mathbf{z}(t) \in \mathbb{R}^{l}$ is the vector of unmeasured outputs and $\mathbf{w}(t)$ is noise signal which belongs to $L_{2}(0, \infty] . \mathbf{A}_{i}, \mathbf{B}_{i}, \mathbf{C}_{i}, \mathbf{D}_{i}, \mathbf{E}_{1 i}, \mathbf{E}_{2 i}, \mathbf{F}_{i}, \mathbf{G}_{i}, \mathbf{L}_{i}, \mathbf{M}_{i}, \mathbf{N}_{i}$ are matrices with appropriate dimension. $\Delta \mathbf{A}_{i}, \Delta \mathbf{B}_{i}, \Delta \mathbf{C}_{i}, \Delta \mathbf{D}_{i}, \Delta \mathbf{E}_{1 i}, \Delta \mathbf{E}_{2 i}$,
$\Delta \mathbf{F}_{i}, \Delta \mathbf{G}_{i}$ are unknown matrices which represent the timevarying uncertainties of the system. $\mathbf{h}(t) \in \mathbb{R}^{q}$ is the health parameters of system, which represents the physical characteristics of each component.

Remark 1. The health parameters are considered in this paper, because the performance degradation of each component in the system is inevitable during the 
working process. And the system's performance is affected by the level of degradation, which is generally described in terms of unmeasured health parameters such as efficiencies and capacities related to each major module in most cases. If the health parameters move away from their nominal values, the shift in other performance variables will be induced. They may be treated as a set of biases and can be augmented to the system states.

It is assumed that both sensors and actuators are timedriven. The data has timestamp and is transmitted in a single-packet, and incorrect order of the data packet does not exist. Since time-delay is related to the bus load at a certain moment, it is assumed that the time-delay has an upper bound, which is $\tau(t) \leq \tau_{m}$ (under the above assumption, the upper bound of time-delay can be estimated; see [23] in detail). As a consequence, $\tau(t)$ can be modeled as a finite state Markov stochastic process on a finite set $\Lambda=\left\{1,2, \ldots, \tau_{m}\right\}$. The transition probability from $\tau(t)=i$ at time $t$ to $\tau(t)=j(j \neq i)$ at time $t+\Delta t$ is

$$
\operatorname{Pr}(\tau(t+\Delta t)=j \mid \tau(t)=i)= \begin{cases}\pi_{i j} \Delta t+o(\Delta t), & i \neq j, \\ 1+\pi_{i j} \Delta t+o(\Delta t), & i=j,\end{cases}
$$

where $\Delta t>0$ and $\lim _{\Delta t \rightarrow 0}(o(\Delta t) / \Delta t)=0 . \pi_{i j} \geq 0$ is the transition probability rates from $\tau(t)=i$ at time $t$ to $\tau(t)=$ $j(j \neq i)$ at time $t+\Delta t$, and there is $\sum_{j=1, j \neq i}^{\tau_{m}} \pi_{i j}=-\pi_{i i}$.

The state feedback controller is applied in this paper, which is $\mathbf{u}(t)=\mathbf{K}_{c} \mathbf{x}(t)$. Then, by fuzzy blending, the global fuzzy augmented model can be obtained as follows:

$$
\left\{\begin{array}{l}
\dot{x}_{h}(t)=\sum_{i=1}^{r} h_{i}(f(t))\left[\left(\mathbf{A}_{h i}+\Delta \mathbf{A}_{h i}\right) \mathbf{x}_{h}(t)+\left(\mathbf{B}_{h i}+\Delta \mathbf{B}_{h i}\right) \mathbf{x}_{h}\right. \\
\left.\cdot(t-\tau(t))+\left(\mathbf{E}_{h 1 i}+\Delta \mathbf{E}_{h 1 i}\right) \mathbf{w}(t)\right], \\
\mathbf{y}(t)=\sum_{i=1}^{r} h_{i}(f(t))\left[\left(\mathbf{C}_{h i}+\Delta \mathbf{C}_{h i}\right) \mathbf{x}_{h}(t)+\left(\mathbf{D}_{h i}+\Delta \mathbf{D}_{h i}\right) \mathbf{x}_{h}\right. \\
\left.\cdot(t-\tau(t))+\left(\mathbf{E}_{2 i}+\Delta \mathbf{E}_{2 i}\right) \mathbf{w}(t)\right], \\
\mathbf{z}(t)=\sum_{i=1}^{r} h_{i}(f(t))\left[\left(\mathbf{F}_{h i}+\Delta \mathbf{F}_{h i}\right) \mathbf{x}_{h}(t)+\left(\mathbf{G}_{h i}+\Delta \mathbf{G}_{h i}\right)\right. \\
\left.\cdot \mathbf{x}_{h}(t-\tau(t))\right], \\
\mathbf{x}_{h}(t)=\left[\begin{array}{c}
\phi(t) \\
0
\end{array}\right], \\
t \in\left[-\tau_{m}, 0\right],
\end{array}\right.
$$

where

$$
\begin{aligned}
\mathbf{x}(t) & =\left[\begin{array}{c}
\mathbf{x}(t) \\
\mathbf{h}(t)
\end{array}\right], \\
\mathbf{A}_{h i} & =\left[\begin{array}{cc}
\mathbf{A}_{i} & \mathbf{L}_{i} \\
0 & \mathbf{I}
\end{array}\right], \\
\mathbf{B}_{h i} & =\left[\begin{array}{cc}
\mathbf{B}_{i} \mathbf{K}_{c} & 0 \\
0 & 0
\end{array}\right], \\
\Delta \mathbf{A}_{h i} & =\left[\begin{array}{cc}
\Delta \mathbf{A}_{i} & 0 \\
0 & 0
\end{array}\right], \\
\Delta \mathbf{B}_{h i} & =\left[\begin{array}{cc}
\Delta \mathbf{B}_{i} \mathbf{K}_{c} & 0 \\
0 & 0
\end{array}\right], \\
\mathbf{E}_{h 1 i} & =\left[\begin{array}{c}
\mathbf{E}_{1 i} \\
0
\end{array}\right], \\
\mathbf{C}_{h i} & =\left[\begin{array}{ll}
\mathbf{C}_{i} & \mathbf{M}_{i}
\end{array}\right], \\
\Delta \mathbf{C}_{h i} & =\left[\begin{array}{ll}
\Delta \mathbf{C}_{i} & 0
\end{array}\right], \\
\Delta \mathbf{E}_{h 1 i} & =\left[\begin{array}{c}
\Delta \mathbf{E}_{1 i} \\
0
\end{array}\right], \\
\mathbf{D}_{h i} & =\left[\begin{array}{ll}
\mathbf{D}_{i} \mathbf{K}_{c} & 0
\end{array}\right], \\
\Delta \mathbf{D}_{h i} & =\left[\begin{array}{ll}
\Delta \mathbf{D}_{i} \mathbf{K}_{c} & 0
\end{array}\right], \\
\mathbf{E}_{h 2 i} & =\mathbf{E}_{2 i}, \\
\Delta \mathbf{E}_{h 2 i} & =\Delta \mathbf{E}_{2 i}, \\
\mathbf{F}_{h i} & =\left[\begin{array}{ll}
\mathbf{F}_{i} & \mathbf{N}_{i}
\end{array}\right], \\
\mathbf{G}_{h i} & =\left[\begin{array}{ll}
\mathbf{G}_{i} \mathbf{K}_{c} & 0
\end{array}\right], \\
\Delta \mathbf{F}_{h i} & =\left[\begin{array}{ll}
\Delta \mathbf{F}_{i} & 0
\end{array}\right], \\
\Delta \mathbf{G}_{h i} & =\left[\begin{array}{ll}
\Delta \mathbf{G}_{i} \mathbf{K}_{c} & 0
\end{array}\right], \\
\vartheta_{i}(f(f) & =\frac{\left.\sum_{i=1}^{r} \vartheta_{i}(f)\right)}{(f(t))},
\end{aligned}
$$

For the underdetermined estimation problem, a lowdimensional model tuning parameter vector $\mathbf{q}(t)$ is produced to represent the information of high-dimensional health parameter vector $\mathbf{h}(t)$. The model tuning parameter vector $\mathbf{q}(t)$ is constructed as a linear combination of all health parameters, given by

$$
\mathbf{q}(t)=\mathbf{V}^{*} \mathbf{h}(t),
$$


where $\mathbf{h}(t) \in \mathbb{R}^{q}, \mathbf{q}(t) \in \mathbb{R}^{k}, k<q$, and $\mathbf{V}^{*}$ is a $k \times q$ transformation matrix, which is applied to construct the tuning parameter vector. The selection of optimal $\mathbf{V}^{*}$ is obtained in Section 4.

Thus, the estimation of the health parameters $\widehat{\mathbf{h}}$ can be obtained as

$$
\widehat{\mathbf{h}}=\mathbf{V}^{* \dagger} \mathbf{q},
$$

where $\mathbf{V}^{* \dagger}$ is the pseudoinverse of $\mathbf{V}^{*}$.

The final augmented dynamic fuzzy model can be rewritten as

$$
\left\{\begin{array}{l}
\dot{x}_{q}(t)=\sum_{i=1}^{r} h_{i}(f(t))\left[\left(\mathbf{A}_{q i}+\Delta \mathbf{A}_{q i}\right) \mathbf{x}_{q}(t)+\left(\mathbf{B}_{q i}+\Delta \mathbf{B}_{q i}\right) \mathbf{x}_{q}(t-\tau(t))+\left(\mathbf{E}_{q 1 i}+\Delta \mathbf{E}_{q 1 i}\right) \mathbf{w}(t)\right], \\
\mathbf{y}(t)=\sum_{i=1}^{r} h_{i}(f(t))\left[\left(\mathbf{C}_{q i}+\Delta \mathbf{C}_{q i}\right) \mathbf{x}_{q}(t)+\left(\mathbf{D}_{q i}+\Delta \mathbf{D}_{q i}\right) \mathbf{x}_{q}(t-\tau(t))+\left(\mathbf{E}_{2 i}+\Delta \mathbf{E}_{2 i}\right) \mathbf{w}(t)\right], \\
\mathbf{z}(t)=\sum_{i=1}^{r} h_{i}(f(t))\left[\left(\mathbf{F}_{q i}+\Delta \mathbf{F}_{q i}\right) \mathbf{x}_{q}(t)+\left(\mathbf{G}_{q i}+\Delta \mathbf{G}_{q i}\right) \mathbf{x}_{q}(t-\tau(t))\right], \\
\mathbf{x}_{q}(t)=\left[\begin{array}{c}
\phi(t) \\
0
\end{array}\right], \\
t \in\left[-\tau_{m}, 0\right],
\end{array}\right.
$$

where

$$
\begin{aligned}
\mathbf{x}_{q}(t) & =\left[\begin{array}{l}
\mathbf{x}(t) \\
\mathbf{q}(t)
\end{array}\right], \\
\mathbf{A}_{q i} & =\left[\begin{array}{cc}
\mathbf{A}_{i} & \mathbf{L}_{i} \mathbf{V}^{* \dagger} \\
0 & \mathbf{I}
\end{array}\right], \\
\mathbf{E}_{q 1 i} & =\left[\begin{array}{c}
\mathbf{E}_{1 i} \\
0
\end{array}\right], \\
\Delta \mathbf{A}_{q i} & =\left[\begin{array}{cc}
\Delta \mathbf{A}_{i} & 0 \\
0 & 0
\end{array}\right], \\
\mathbf{B}_{q i} & =\left[\begin{array}{cc}
\mathbf{B}_{i} \mathbf{K}_{c} & 0 \\
0 & 0
\end{array}\right], \\
\Delta \mathbf{B}_{q i} & =\left[\begin{array}{cc}
\Delta \mathbf{B}_{i} \mathbf{K}_{c} & 0 \\
0 & 0
\end{array}\right], \\
\Delta \mathbf{E}_{q 1 i} & =\left[\begin{array}{cc}
\Delta \mathbf{E}_{1 i} \\
0
\end{array}\right], \\
\mathbf{C}_{q i} & =\left[\begin{array}{ll}
\mathbf{C}_{i} \mathbf{M}_{i} \mathbf{V}^{* \dagger}
\end{array}\right], \\
\Delta \mathbf{C}_{q i} & =\left[\begin{array}{ll}
\Delta \mathbf{C}_{i} & 0
\end{array}\right], \\
\mathbf{D}_{q i} & =\left[\begin{array}{ll}
\mathbf{D}_{i} \mathbf{K}_{c} & 0
\end{array}\right], \\
\Delta \mathbf{D}_{q i} & =\left[\begin{array}{ll}
\Delta \mathbf{D}_{i} \mathbf{K}_{c} & 0
\end{array}\right], \\
\mathbf{E}_{q 2 i} & =\mathbf{E}_{2 i}, \\
\Delta \mathbf{E}_{q 2 i} & =\Delta \mathbf{E}_{2 i}, \\
\mathbf{F}_{q i} & =\left[\begin{array}{ll}
\mathbf{F}_{i} \mathbf{N}_{i} \mathbf{V}^{* \dagger}
\end{array}\right], \\
\mathbf{G}_{q i} & =\left[\begin{array}{ll}
\mathbf{G}_{i} \mathbf{K}_{c} & 0
\end{array}\right], \\
\Delta \mathbf{F}_{q i} & =\left[\begin{array}{ll}
\Delta \mathbf{F}_{i} & 0
\end{array}\right], \\
\Delta \mathbf{G}_{q i} & =\left[\begin{array}{ll}
\Delta \mathbf{G}_{i} \mathbf{K}_{c} & 0
\end{array}\right],
\end{aligned}
$$

Remark 2. If the model tuning parameter vector is of appropriate dimension, the augmented system (7) is observable and the observer-based filter can be used to estimate the unknown health and performance parameters. And the key of the estimation is to find the optimal transformation matrix so that the low dimension tuning vector can represent as much of the information as possible. Moreover, different from the method in [17-19], the solution of the optimal transformation matrix is transformed into an optimization problem.

It is assumed that the uncertainties of the system can be described in the following form:

$$
\left[\begin{array}{cccc}
\Delta \mathbf{A}_{q i} & \Delta \mathbf{B}_{q i} & \Delta \mathbf{E}_{q 1 i} & \Delta \mathbf{F}_{q i}^{T} \\
\Delta \mathbf{C}_{q i} & \Delta \mathbf{D}_{q i} & \Delta \mathbf{E}_{q 2 i} & \Delta \mathbf{G}_{q i}^{T}
\end{array}\right]=\left[\begin{array}{c}
\mathbf{U}_{1 i} \\
\mathbf{U}_{2 i}
\end{array}\right] \mathbf{S}_{i}\left[\begin{array}{llll}
\mathbf{V}_{1 i} & \mathbf{V}_{2 i} & \mathbf{V}_{3 i} & \mathbf{V}_{4 i}
\end{array}\right],
$$

where $\mathbf{U}_{1 i}, \mathbf{U}_{2 i}, \mathbf{V}_{1 i}, \mathbf{V}_{2 i}, \mathbf{V}_{3 i}, \mathbf{V}_{4 i}$ are known matrices and $\mathbf{S}_{i}$ is a time-varying unknown matrix which satisfies

$$
\mathbf{S}_{i}^{T} \mathbf{S}_{i} \leq \mathbf{I} .
$$

Then the following observer-based fuzzy filter is constructed:

$$
\begin{aligned}
\dot{\hat{\mathbf{x}}}(t)= & \sum_{i=1}^{r} h_{i}(f(t))\left[\left(\mathbf{A}_{q i}+\Delta \mathbf{A}_{q i}\right) \widehat{\mathbf{x}}(t)\right. \\
& \left.+\left(\mathbf{B}_{q i}+\Delta \mathbf{B}_{q i}\right) \widehat{\mathbf{x}}(t-\tau(t))+\mathbf{K}_{i}(\mathbf{y}(t)-\widehat{\mathbf{y}}(t))\right], \\
\widehat{\mathbf{y}}(t)= & \sum_{i=1}^{r} h_{i}(f(t))\left[\left(\mathbf{C}_{q i}+\Delta \mathbf{C}_{q i}\right) \widehat{\mathbf{x}}(t)+\left(\mathbf{D}_{q i}+\Delta \mathbf{D}_{q i}\right) \widehat{\mathbf{x}}(t-\tau(t))\right], \\
\widehat{\mathbf{z}}(t)= & \sum_{i=1}^{r} h_{i}(f(t))\left[\left(\mathbf{F}_{q i}+\Delta \mathbf{F}_{q i}\right) \widehat{\mathbf{x}}(t)+\left(\mathbf{G}_{q i}+\Delta \mathbf{G}_{q i}\right) \widehat{\mathbf{x}}(t-\tau(t))\right],
\end{aligned}
$$


where $\widehat{\mathbf{x}}(t)$ is the filter state, and $\widehat{\mathbf{y}}(t)$ and $\widehat{\mathbf{z}}(t)$ are the filter outputs. $\mathbf{K}_{i}$ is the filter parameter to be determined.

The estimation error of state is

$$
\begin{aligned}
\tilde{x}(t)= & \mathbf{x}_{q}(t)-\widehat{x}(t) \\
= & \left(\mathbf{A}_{q i}-\mathbf{K}_{i} \mathbf{C}_{q i}+\Delta \mathbf{A}_{q i}-\mathbf{K}_{i} \Delta \mathbf{C}_{q i}\right) \tilde{x}(t) \\
& +\left(\mathbf{B}_{q i}-\mathbf{K}_{i} \mathbf{D}_{q i}+\Delta \mathbf{B}_{q i}-\mathbf{K}_{i} \Delta \mathbf{D}_{q i}\right) \tilde{x}(t-\tau(t)) \\
& +\left(\mathbf{E}_{q 1 i}-\mathbf{K}_{i} \mathbf{E}_{2 i}+\Delta \mathbf{E}_{q 1 i}-\mathbf{K}_{i} \Delta \mathbf{E}_{2 i}\right) \mathbf{w}(t) .
\end{aligned}
$$

Define $\boldsymbol{\xi}(t)=\left[\begin{array}{lll}\mathbf{x}_{q}^{T} & \tilde{x}^{T}(t)\end{array}\right]^{T}$ and $\mathbf{e}(t)=\mathbf{z}(t)-\widehat{z}(t)$, and the filtering error system is given by

$$
\begin{aligned}
\dot{\xi}(t)= & \sum_{i=1}^{r} h_{i}(f(t))\left[\left(\bar{A}_{i}+\Delta \bar{A}_{i}\right) \xi(t)+\left(\bar{B}_{i}+\Delta \bar{B}_{i}\right) \boldsymbol{\xi}(t-\tau(t))\right. \\
& \left.+\left(\bar{E}_{i}+\Delta \bar{E}_{i}\right) \mathbf{w}(t)\right], \\
\dot{\mathbf{e}}(t)= & \sum_{i=1}^{r} h_{i}(f(t))\left[\left(\bar{F}_{i}+\Delta \bar{F}_{i}\right) \xi(t)+\left(\bar{G}_{i}+\Delta \bar{G}_{i}\right) \xi(t-\tau(t))\right],
\end{aligned}
$$

where

$$
\begin{aligned}
\overline{\mathbf{A}}_{i} & =\left[\begin{array}{cc}
\mathbf{A}_{q i} & 0 \\
0 & \mathbf{A}_{q i}-\mathbf{K}_{i} \mathbf{C}_{q i}
\end{array}\right], \\
\overline{\mathbf{B}}_{i} & =\left[\begin{array}{cc}
\mathbf{B}_{q i} & 0 \\
0 & \mathbf{B}_{q i}-\mathbf{K}_{i} \mathbf{D}_{q i}
\end{array}\right], \\
\Delta \overline{\mathbf{A}}_{i} & =\left[\begin{array}{cc}
\Delta \mathbf{A}_{q i} & 0 \\
0 & \Delta \mathbf{A}_{q i}-\mathbf{K}_{i} \Delta \mathbf{C}_{q i}
\end{array}\right], \\
\overline{\mathbf{E}}_{i} & =\left[\begin{array}{c}
\mathbf{E}_{q 1 i} \\
\mathbf{E}_{q 1 i}-\mathbf{K}_{i} \mathbf{E}_{2 i}
\end{array}\right], \\
\Delta \overline{\mathbf{B}}_{i} & =\left[\begin{array}{cc}
\Delta \mathbf{B}_{q i} & 0 \\
0 & \Delta \mathbf{B}_{q i}-\mathbf{K}_{i} \Delta \mathbf{D}_{q i}
\end{array}\right], \\
\Delta \overline{\mathbf{E}}_{i} & =\left[\begin{array}{cc}
\Delta & \Delta \mathbf{E}_{q 1 i} \\
\Delta \mathbf{E}_{q 1 i}-\mathbf{K}_{i} \Delta \mathbf{E}_{2 i}
\end{array}\right], \\
\overline{\mathbf{F}}_{i} & =\left[\begin{array}{ll}
0 & \mathbf{F}_{q i}
\end{array}\right], \\
\Delta \overline{\mathbf{F}}_{i} & =\left[\begin{array}{ll}
0 & \Delta \mathbf{F}_{q i}
\end{array}\right], \\
\overline{\mathbf{G}}_{i} & =\left[\begin{array}{ll}
0 & \mathbf{G}_{q i}
\end{array}\right], \\
\Delta \overline{\mathbf{G}}_{i} & =\left[\begin{array}{ll}
0 & \Delta \mathbf{G}_{q i}
\end{array}\right] .
\end{aligned}
$$

Define the following parameters:

$$
\begin{aligned}
\overline{\mathbf{A}} & =\sum_{i=1}^{r} h_{i}(f(t)) \overline{\mathbf{A}}_{i}, \\
\Delta \overline{\mathbf{A}} & =\sum_{i=1}^{r} h_{i}(f(t)) \Delta \overline{\mathbf{A}}_{i}, \\
\overline{\mathbf{B}} & =\sum_{i=1}^{r} h_{i}(f(t)) \overline{\mathbf{B}}_{i}, \\
\Delta \overline{\mathbf{B}} & =\sum_{i=1}^{r} h_{i}(f(t)) \Delta \overline{\mathbf{B}}_{i}, \\
\overline{\mathbf{E}} & =\sum_{i=1}^{r} h_{i}(f(t)) \overline{\mathbf{E}}_{i}, \\
\Delta \overline{\mathbf{E}} & =\sum_{i=1}^{r} h_{i}(f(t)) \Delta \overline{\mathbf{E}}_{i}, \\
\overline{\mathbf{F}} & =\sum_{i=1}^{r} h_{i}(f(t)) \overline{\mathbf{F}}_{i}, \\
\Delta \overline{\mathbf{F}} & =\sum_{i=1}^{r} h_{i}(f(t)) \Delta \overline{\mathbf{F}}_{i}, \\
\overline{\mathbf{G}} & =\sum_{i=1}^{r} h_{i}(f(t)) \overline{\mathbf{G}}_{i}, \\
& =\sum_{i=1}^{r} h_{i}(f(t)) \Delta \overline{\mathbf{G}}_{i} .
\end{aligned}
$$

The goal is to design a filter in form of (11) so that the filtering error system can meet the following requirements simultaneously:

(1) The filtering error system is asymptotically stable when $\mathbf{w}(t)=0$

(2) Under the zero initial condition, the filtering error system satisfies

$$
\|\mathbf{e}(t)\|_{2} \leq \gamma\|\mathbf{w}(t)\|_{2},
$$

for any nonzero $\mathbf{w}(t) \in L_{2}(0, \infty]$.

\section{Main Results}

In this section, the sufficient condition for the existence of the desired filter is derived in terms of LMIs solutions. Before proceeding with the study, the following Lemma is needed.

Lemma 1 (see [24]). D, E, F are real matrices with appropriate dimensions, and $\mathbf{F}$ is a time-varying unknown matrix which satisfies $\mathbf{F}^{T} \mathbf{F} \leq \mathbf{I}$. Then for a scalar $\varepsilon>0$, the following inequality 


$$
\mathbf{D F} \quad \mathbf{E}+\mathbf{E}^{T} \mathbf{F}^{T} \mathbf{D}^{T} \leq \varepsilon^{-1} \mathbf{D} \mathbf{D}^{T}+\varepsilon \mathbf{E}^{T} \mathbf{E},
$$

always holds.
Theorem 1. If there exist positive matrices $\mathbf{P}>0, \mathbf{Q}>0, \mathbf{R}>0$, and matrix $\mathbf{W}$ such that the following inequality holds:

$$
\bar{\psi}=\left[\begin{array}{cccccc}
\Gamma & \mathbf{P}(\overline{\mathbf{B}}+\Delta \overline{\mathbf{B}})+\mathbf{W} & \mathbf{P}(\overline{\mathbf{E}}+\Delta \overline{\mathbf{E}}) & \tau_{m} \mathbf{W} & \tau_{m}(\overline{\mathbf{A}}+\Delta \overline{\mathbf{A}})^{T} \mathbf{R} & (\overline{\mathbf{F}}+\Delta \overline{\mathbf{F}})^{T} \\
* & -\mathbf{Q} & 0 & 0 & \tau_{m}(\overline{\mathbf{B}}+\Delta \overline{\mathbf{B}})^{T} \mathbf{R} & (\overline{\mathbf{G}}+\Delta \overline{\mathbf{G}})^{T} \\
* & * & -\gamma^{2} \mathbf{I} & 0 & \tau_{m}(\overline{\mathbf{E}}+\Delta \overline{\mathbf{E}})^{T} \mathbf{R} & 0 \\
* & * & * & -\tau_{m} \mathbf{R} & 0 & 0 \\
* & * & * & * & -\tau_{m} \mathbf{R} & 0 \\
* & * & * & * & * & -\mathbf{I}
\end{array}\right]<0
$$

where

$$
\Gamma=\mathbf{P}(\overline{\mathbf{A}}+\Delta \overline{\mathbf{A}})+(\overline{\mathbf{A}}+\Delta \overline{\mathbf{A}})^{T} \mathbf{P}+\mathbf{Q}-\mathbf{W}-\mathbf{W}^{T},
$$

then the filtering error system is asymptotically stable and the prescribed Ho performance $\gamma$ can be guaranteed.

Proof. Select the Lyapunov function as

$$
V(t)=V_{1}(t)+V_{2}(t)+V_{3}(t),
$$

where

$$
\begin{aligned}
& V_{1}(t)=\xi^{T}(t) \mathbf{P} \xi(t), \\
& V_{2}(t)=\int_{t-\tau(t)}^{t} \xi^{T}(\alpha) \mathbf{Q} \xi(\alpha) \mathrm{d} \alpha, \\
& V_{3}(t)=\int_{-\tau_{m}}^{0} \int_{t+\beta}^{t} \dot{\xi}^{T}(\alpha) \mathbf{R} \dot{\xi}(\alpha) \mathrm{d} \alpha \mathrm{d} \beta .
\end{aligned}
$$

When $w(t)=0$, the derivation of $V(t)$ is

$\dot{V}_{1}(t)=2 \boldsymbol{\xi}^{T}(t) \mathbf{P}((\overline{\mathbf{A}}+\Delta \overline{\mathbf{A}}) \boldsymbol{\xi}(t)+(\overline{\mathbf{B}}+\Delta \overline{\mathbf{B}}) \boldsymbol{\xi}(t-\tau(t)))$,

$\dot{V}_{2}(t) \leq \xi^{T}(t) \mathbf{Q} \xi(t)-\xi^{T}(t-\tau(t)) \mathbf{Q} \xi(t-\tau(t))$,

$\dot{V}_{3}(t) \leq \tau_{m} \dot{\xi}^{T}(t) \mathbf{R} \dot{\xi}(t)-\int_{t-\tau(t)}^{t} \dot{\xi}^{T}(\alpha) \mathbf{R} \dot{\xi}(\alpha) \mathrm{d} \alpha$.

It is obvious that there is

$$
\xi(t-\tau(t))=\xi(t)-\int_{t-\tau(t)}^{t} \dot{\xi}^{T}(\alpha) \mathrm{d} \alpha .
$$

Thus, $V(t)$ can be further rewritten as

$$
\begin{aligned}
\dot{V}(t) \leq & 2 \xi^{T}(t) \mathbf{P}((\overline{\mathbf{A}}+\Delta \overline{\mathbf{A}}) \xi(t)+(\overline{\mathbf{B}}+\Delta \overline{\mathbf{B}}) \xi(t-\tau(t)))+\xi^{T}(t) \mathbf{Q} \xi(t)-\xi^{T}(t-\tau(t)) \mathbf{Q} \xi(t-\tau(t)) \\
& +\tau_{m} \dot{\xi}^{T}(t) \mathbf{R} \dot{\xi}(t)-\int_{t-\tau(t)}^{t} \dot{\xi}^{T}(\alpha) \mathbf{R} \dot{\xi}(\alpha) \mathrm{d} \alpha+2 \xi^{T}(t) \mathbf{W}\left(\xi^{T}(t-\tau(t))-\xi^{T}(t)+\int_{t-\tau(t)}^{t} \dot{\xi}^{T}(\alpha) \mathrm{d} \alpha\right) \\
& \leq \frac{1}{\tau(t)} \int_{t-\tau(t)}^{t}\left[\begin{array}{c}
\xi(t) \\
\xi(t-\tau(t)) \\
\dot{\xi}(\alpha)
\end{array}\right]^{T} \Psi_{0}\left[\begin{array}{c}
\xi(t) \\
\xi(t-\tau(t)) \\
\dot{\xi}(\alpha)
\end{array}\right],
\end{aligned}
$$

where

$$
\Psi_{0}=\left(\left[\begin{array}{ccc}
\Gamma & \mathbf{P}(\overline{\mathbf{B}}+\Delta \overline{\mathbf{B}})+\mathbf{W} & \tau_{m} \mathbf{W} \\
* & -\mathbf{Q} & 0 \\
* & * & -\tau_{m} \mathbf{R}
\end{array}\right]+\tau_{m}\left[\begin{array}{c}
(\overline{\mathbf{A}}+\Delta \overline{\mathbf{A}})^{T} \\
(\overline{\mathbf{B}}+\Delta \overline{\mathbf{B}})^{T} \\
0
\end{array}\right] \mathbf{R}\left[\begin{array}{c}
(\overline{\mathbf{A}}+\Delta \overline{\mathbf{A}})^{T} \\
(\overline{\mathbf{B}}+\Delta \overline{\mathbf{B}})^{T} \\
0
\end{array}\right]^{T}\right)
$$

By the Schur complement, if the inequality (24) holds, it can be obtained that there is $\Psi_{0}<0$. Thus, there is $\dot{V}(t)<0$ and the filtering error system is asymptotically stable.

Secondly, define a new function

$$
J=\int_{0}^{T}\left[\mathbf{e}^{T}(t) \mathbf{e}(t)-\gamma^{2} \mathbf{w}^{T}(t) \mathbf{w}(t)\right] \mathrm{d} t,
$$

where $T$ is a scalar which satisfies $T>0$.

Thus, under the zero initial condition, there is 


$$
\begin{aligned}
J & =\int_{0}^{T}\left[\mathbf{e}^{T}(t) \mathbf{e}(t)-\gamma^{2} \mathbf{w}^{T}(t) \mathbf{w}(t)+\dot{V}(t)\right] \mathrm{d} t-V(T) \\
& \leq \int_{0}^{T}\left[\mathbf{e}^{T}(t) \mathbf{e}(t)-\gamma^{2} \mathbf{w}^{T}(t) \mathbf{w}(t)+\dot{V}(t)\right] \mathrm{d} t \\
& \leq\left[\begin{array}{c}
\xi(t) \\
\xi(t-\tau(t)) \\
w(t) \\
\dot{\xi}(\alpha)
\end{array}\right] \psi_{1}\left[\begin{array}{c}
\xi(t-\tau(t)) \\
w(t) \\
\dot{\xi}(\alpha)
\end{array}\right],
\end{aligned}
$$

where

$$
\begin{aligned}
\boldsymbol{\psi}_{1}= & {\left[\begin{array}{cccc}
\Gamma & \mathbf{P}(\bar{B}+\Delta \bar{B})+\mathbf{W} & \mathbf{P}(\bar{E}+\Delta \bar{E}) & \tau_{m} \mathbf{W} \\
* & -\mathbf{Q} & 0 & 0 \\
* & * & -\gamma^{2} \mathbf{I} & 0 \\
* & * & * & -\tau_{m} \mathbf{R}
\end{array}\right] } \\
& +\tau_{m}\left[\begin{array}{c}
(\bar{A}+\Delta \bar{A})^{T} \\
(\bar{B}+\Delta \bar{B})^{T} \\
(\bar{E}+\Delta \bar{E})^{T} \\
0
\end{array}\right] \mathbf{R}\left[\begin{array}{c}
(\bar{A}+\Delta \bar{A})^{T} \\
(\bar{B}+\Delta \bar{B})^{T} \\
(\bar{E}+\Delta \bar{E})^{T} \\
0
\end{array}\right] \\
& +\left[\begin{array}{c}
(\bar{F}+\Delta \bar{F})^{T} \\
(\bar{G}+\Delta \bar{G})^{T} \\
0 \\
0
\end{array}\right]\left[\begin{array}{c}
(\bar{F}+\Delta \bar{F})^{T} \\
(\bar{G}+\Delta \bar{G})^{T} \\
0 \\
0
\end{array}\right] .
\end{aligned}
$$

According to the Schur complement, it follows that

$$
\psi_{1}<0 \Leftrightarrow \bar{\psi}<0 \text {. }
$$

Thus, if the inequality $\bar{\psi}<0$ holds, there is $J<0$ and equation (16) holds for any nonzero $\mathbf{w}(t) \in L_{2}[0, \infty]$. The proof is completed.

Theorem 2. For a given scalar $\gamma>0$, the filtering problem is solvable under the conditions above with a Ho performance level $\gamma$, if there exist positive matrices $\mathbf{P}_{1}, \mathbf{P}_{2}, \mathbf{Q}_{1}, \mathbf{Q}_{2}, \mathbf{R}_{1}, \mathbf{R}_{2}$, matrices $\mathbf{W}_{1}, \mathbf{W}_{2}, \mathbf{Z}_{i}$ and scalars $\varepsilon_{1 i}, \varepsilon_{2 i}, \varepsilon_{3 i j}, \varepsilon_{4 i}, \varepsilon_{5 i}>0(1 \leq i \leq j \leq r)$ such that the following inequalities hold:

$$
\begin{aligned}
& \boldsymbol{\Omega}_{i i}<0, \quad i=1,2, \ldots, r \\
& \boldsymbol{\Omega}_{i j}+\boldsymbol{\Omega}_{j i}<0, \quad i<j, i, j=1,2, \ldots, r,
\end{aligned}
$$

where

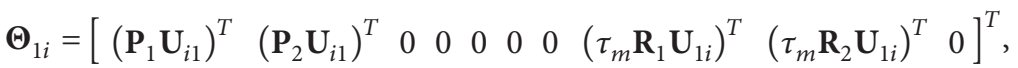

$$
\begin{aligned}
& \boldsymbol{\Xi}_{1 i}=\left[\begin{array}{llllllllll}
\mathbf{V}_{1 i} & 0 & \mathbf{V}_{2 i} & 0 & \mathbf{V}_{3 i} & 0 & 0 & 0 & 0 & 0
\end{array}\right] \text {, } \\
& \boldsymbol{\Theta}_{2 i}=\left[\begin{array}{lllllllllll}
0 & \left(\mathbf{P}_{2} \mathbf{U}_{1 i}\right)^{T} & 0 & 0 & 0 & 0 & 0 & 0 & \left(\tau_{m} \mathbf{R}_{2} \mathbf{U}_{1 i}\right)^{T} & 0
\end{array}\right]^{T}, \\
& \boldsymbol{\Xi}_{2 i}=\left[\begin{array}{llllllllll}
0 & \mathbf{V}_{1 i} & 0 & \mathbf{V}_{2 i} & 0 & 0 & 0 & 0 & 0 & 0
\end{array}\right] \text {, } \\
& \boldsymbol{\Theta}_{3 i j}=\left[\begin{array}{lllllllllll}
0 & \left(\mathbf{P}_{2} \mathbf{K}_{i} \mathbf{U}_{2 i}\right)^{T} & 0 & 0 & 0 & 0 & 0 & 0 & \left(\tau_{m} \mathbf{R}_{2} \mathbf{K}_{i} \mathbf{U}_{2 i}\right)^{T} & 0
\end{array}\right]^{T}, \\
& \boldsymbol{\Xi}_{3 i}=\left[\begin{array}{llllllllll}
0 & \mathbf{V}_{1 i} & 0 & \mathbf{V}_{2 i} & \mathbf{V}_{3 i} & 0 & 0 & 0 & 0 & 0
\end{array}\right] \text {, } \\
& \boldsymbol{\Theta}_{4 i}=\left[\begin{array}{llllllllll}
0 & 0 & 0 & 0 & 0 & 0 & 0 & 0 & 0 & \mathbf{U}_{1 i}^{T}
\end{array}\right]^{T}, \\
& \boldsymbol{\Xi}_{4 i}=\left[\begin{array}{llllllllll}
0 & \mathbf{V}_{4 i} & 0 & 0 & 0 & 0 & 0 & 0 & 0 & 0
\end{array}\right], \\
& \boldsymbol{\Theta}_{5 i}=\left[\begin{array}{llllllllll}
0 & 0 & 0 & 0 & 0 & 0 & 0 & 0 & 0 & \mathbf{U}_{2 i}^{T}
\end{array}\right]^{T}, \\
& \boldsymbol{\Xi}_{5 i}=\left[\begin{array}{llllllllll}
0 & 0 & 0 & \mathbf{V}_{4 i} & 0 & 0 & 0 & 0 & 0 & 0
\end{array}\right] \text {, } \\
& \Gamma_{11}=\mathbf{P}_{1} \mathbf{A}_{q i}+\mathbf{A}_{q i}^{T} \mathbf{P}_{1}+\mathbf{Q}_{1}-\mathbf{W}_{1}-\mathbf{W}_{1}^{T}, \\
& \Gamma_{22}=\mathbf{P}_{2} \mathbf{A}_{q i}-\mathbf{Z}_{1 i} \mathbf{C}_{q i}+\mathbf{A}_{q i}^{T} \mathbf{P}_{2}-\mathbf{C}_{q i}^{T} \mathbf{Z}_{1 i}^{T}+\mathbf{Q}_{2}-\mathbf{W}_{2}-\mathbf{W}_{2}^{T}, \\
& \Gamma_{13}=\mathbf{P}_{1} \mathbf{B}_{q i}+\mathbf{W}_{1} \text {, } \\
& \Gamma_{24}=\mathbf{P}_{2} \mathbf{B}_{q i}-\mathbf{Z}_{1 i} \mathbf{D}_{q i}+\mathbf{W}_{2} \text {, } \\
& \Gamma_{25}=\mathbf{P}_{2} \mathbf{E}_{q 1 i}-\mathbf{Z}_{1 i} \mathbf{E}_{2 i} \text {, } \\
& \boldsymbol{\Omega}_{i j}=\left[\begin{array}{cccccc}
\boldsymbol{\Omega}_{0 i j}+\boldsymbol{\varepsilon}_{1 i} \boldsymbol{\Xi}_{1 i}^{T} \boldsymbol{\Xi}_{1 i}+\varepsilon_{2 i} \boldsymbol{\Xi}_{2 i}^{T} \boldsymbol{\Xi}_{2 i}-\varepsilon_{3 i j} \boldsymbol{\Xi}_{3 i}^{T} \boldsymbol{\Xi}_{3 i}+\varepsilon_{4 i} \boldsymbol{\Xi}_{4 i}^{T} \boldsymbol{\Xi}_{4 i}+\varepsilon_{5 i} \boldsymbol{\Xi}_{5 i}^{T} \boldsymbol{\Xi}_{5 i} & \boldsymbol{\Theta}_{1 i} & \boldsymbol{\Theta}_{2 i} & \boldsymbol{\Theta}_{3 i j} & \boldsymbol{\Theta}_{4 i} & \boldsymbol{\Theta}_{5 i} \\
* & -\varepsilon_{1 i} & 0 & 0 & 0 & 0 \\
* & * & -\varepsilon_{1 i 2} & 0 & 0 & 0 \\
* & * & * & \varepsilon_{3 i j} & 0 & 0 \\
* & * & * & * & -\varepsilon_{4 i} & 0 \\
* & * & * & * & * & -\varepsilon_{5 i}
\end{array}\right],
\end{aligned}
$$




$$
\mathbf{\Omega}_{0 i j}=\left[\begin{array}{cccccccccc}
\Gamma_{11} & 0 & \Gamma_{13} & 0 & \mathbf{P}_{1} \mathbf{E}_{q 1 i} & \tau_{m} \mathbf{W}_{1} & 0 & \tau_{m} \mathbf{A}_{q i}^{T} \mathbf{R}_{1} & 0 & 0 \\
* & \Gamma_{22} & 0 & \Gamma_{24} & \Gamma_{25} & 0 & \tau_{m} \mathbf{W}_{2} & 0 & \tau_{m}\left[\mathbf{A}_{q i}^{T} \mathbf{R}_{2}-\mathbf{C}_{q i}^{T} \mathbf{Z}_{2 i}\right] & \mathbf{F}_{q i}^{T} \\
* & * & -\mathbf{Q}_{1} & 0 & 0 & 0 & 0 & \tau_{m} \mathbf{B}_{q i}^{T} \mathbf{R} & 0 & 0 \\
* & * & * & -\mathbf{Q}_{2} & 0 & 0 & 0 & 0 & \tau_{m}\left[\mathbf{B}_{q i}^{T} \mathbf{R}_{2}-\mathbf{D}_{q i}^{T} \mathbf{Z}_{2 i}\right] & \mathbf{G}_{q i}^{T} \\
* & * & * & * & -\gamma^{2} \mathbf{I} & 0 & 0 & \tau_{m} \mathbf{E}_{q 1 i}^{T} \mathbf{R}_{1} & \tau_{m}\left[\mathbf{E}_{q 1 i}^{T} \mathbf{R}_{2}-\mathbf{E}_{2 i}^{T} \mathbf{Z}_{2 i}\right] & 0 \\
* & * & * & * & * & -\tau_{m} \mathbf{R}_{1} & 0 & 0 & 0 & 0 \\
* & * & * & * & * & * & -\tau_{m} \mathbf{R}_{2} & 0 & 0 & 0 \\
* & * & * & * & * & * & * & -\tau_{m} \mathbf{R}_{1} & 0 & 0 \\
* & * & * & * & * & * & * & * & -\tau_{m} \mathbf{R}_{2} & 0 \\
* & * & * & * & * & * & * & * & * & -\mathbf{I}
\end{array}\right],
$$

Moreover, the filter parameter can be solved by

$$
\mathbf{K}_{i}=\mathbf{P}_{2}^{-1} \mathbf{Z}_{1 i}
$$

Proof. Let

$$
\begin{gathered}
\mathbf{P}=\operatorname{diag}\left\{\mathbf{P}_{1}, \mathbf{P}_{2}\right\}, \\
\mathbf{Q}=\operatorname{diag}\left\{\mathbf{Q}_{1}, \mathbf{Q}_{2}\right\}, \\
\mathbf{R}=\operatorname{diag}\left\{\mathbf{R}_{1}, \mathbf{R}_{2}\right\}, \\
\mathbf{W}=\operatorname{diag}\left\{\mathbf{W}_{1}, \mathbf{W}_{2}\right\} .
\end{gathered}
$$

Then $\bar{\psi}$ can be rewritten as

$$
\bar{\psi}=\left[\begin{array}{cccccc}
\Upsilon_{11} & \Upsilon_{12} & \Upsilon_{13} & \tau_{m} \mathbf{W} & \tau_{m} \Upsilon_{15} & (\bar{F}+\Delta \bar{F})^{T} \\
* & -\mathbf{Q} & 0 & 0 & \tau_{m} \Upsilon_{25} & (\bar{G}+\Delta \bar{G})^{T} \\
* & * & -\gamma^{2} \mathbf{I} & 0 & \tau_{m} \Upsilon_{35} & 0 \\
* & * & * & -\tau_{m} \mathbf{R} & 0 & 0 \\
* & * & * & * & -\tau_{m} \mathbf{R} & 0 \\
* & * & * & * & * & -\mathbf{I}
\end{array}\right],
$$

Then, for further analysis, based on equation (9) and Lemma 1, equation (35) can be decomposed into

$$
\begin{aligned}
\bar{\psi}= & \sum_{i=1}^{r} \sum_{j=1}^{r} h_{i}(f(t)) h_{j}(f(t))\left\{\boldsymbol{\Omega}_{0 i j}+\boldsymbol{\Theta}_{1 i} \mathbf{S}_{i} \boldsymbol{\Xi}_{1 i}+\boldsymbol{\Xi}_{1 i}^{T} \mathbf{S}_{i}^{T} \boldsymbol{\Theta}_{1 i}^{T}\right. \\
& +\boldsymbol{\Theta}_{2 i} \mathbf{S}_{i} \boldsymbol{\Xi}_{2 i}+\boldsymbol{\Xi}_{2 i}^{T} \mathbf{S}_{i}^{T} \boldsymbol{\Theta}_{2 i}^{T}-\boldsymbol{\Theta}_{3 i j} \mathbf{S}_{i} \boldsymbol{\Xi}_{3 i}-\boldsymbol{\Xi}_{3 i}^{T} \mathbf{S}_{i}^{T} \boldsymbol{\Theta}_{3 i j}^{T} \\
& \left.+\boldsymbol{\Theta}_{4 i} \mathbf{S}_{i} \boldsymbol{\Xi}_{4 i}+\boldsymbol{\Xi}_{4 i}^{T} \mathbf{S}_{i}^{T} \boldsymbol{\Theta}_{4 i}^{T}+\boldsymbol{\Theta}_{5 i} \mathbf{S}_{i} \boldsymbol{\Xi}_{5 i}+\boldsymbol{\Xi}_{5 i}^{T} \mathbf{S}_{i}^{T} \boldsymbol{\Theta}_{5 i}^{T}\right\} \\
\leq & \sum_{i=1}^{r} \sum_{j=1}^{r} h_{i}(f(t)) h_{j}(f(t))\left\{\boldsymbol{\Omega}_{0 i j}+\frac{1}{\varepsilon_{1 i}} \boldsymbol{\Theta}_{1 i} \boldsymbol{\Theta}_{1 i}^{T}+\varepsilon_{1 i} \boldsymbol{\Xi}_{1 i}^{T} \boldsymbol{\Xi}_{1 i}\right. \\
& +\frac{1}{\varepsilon_{2 i}} \boldsymbol{\Theta}_{2 i} \boldsymbol{\Theta}_{2 i}^{T}+\varepsilon_{2 i} \boldsymbol{\Xi}_{2 i}^{T} \boldsymbol{\Xi}_{2 i}-\frac{1}{\varepsilon_{3 i j}} \boldsymbol{\Theta}_{3 i j} \boldsymbol{\Theta}_{3 i j}^{T}-\varepsilon_{3 i j} \boldsymbol{\Xi}_{3 i}^{T} \boldsymbol{\Xi}_{3 i} \\
& \left.+\frac{1}{\varepsilon_{4 i}} \boldsymbol{\Theta}_{4 i} \boldsymbol{\Theta}_{4 i}^{T}+\varepsilon_{4 i} \boldsymbol{\Xi}_{4 i}^{T} \boldsymbol{\Xi}_{4 i}+\frac{1}{\varepsilon_{5 i}} \boldsymbol{\Theta}_{5 i} \boldsymbol{\Theta}_{5 i}^{T}+\varepsilon_{5 i} \boldsymbol{\Xi}_{5 i}^{T} \boldsymbol{\Xi}_{5 i}\right\}
\end{aligned}
$$

Moreover, define

$$
\begin{aligned}
& \mathbf{Z}_{1 i}=\mathbf{P}_{2} \mathbf{K}_{i}, \\
& \mathbf{Z}_{2 i}=\mathbf{K}_{i}^{T} \mathbf{R}_{2} .
\end{aligned}
$$

$$
\begin{aligned}
\boldsymbol{\delta}_{1}= & \mathbf{P}_{1}\left(\mathbf{A}_{q i}+\Delta \mathbf{A}_{q i}\right)+\left(\mathbf{A}_{q i}+\Delta \mathbf{A}_{q i}\right)^{T} \mathbf{P}_{1}+\mathbf{Q}_{1}-\mathbf{W}_{1}-\mathbf{W}_{1}^{T}, \\
\boldsymbol{\delta}_{2}= & \mathbf{P}_{2}\left(\mathbf{A}_{q i}+\Delta \mathbf{A}_{q i}\right)-\mathbf{P}_{2} \mathbf{K}_{i}\left(\mathbf{C}_{q i}+\Delta \mathbf{C}_{q i}\right)+\left(\mathbf{A}_{q i}+\Delta \mathbf{A}_{q i}\right)^{T} \\
& \cdot \mathbf{P}_{2}-\left(\mathbf{C}_{q i}+\Delta \mathbf{C}_{q i}\right)^{T} \mathbf{K}_{i}^{T} \mathbf{P}_{2}+\mathbf{Q}_{2}-\mathbf{W}_{2}-\mathbf{W}_{2}^{T}, \\
\Upsilon_{12}= & \operatorname{diag}\left\{\mathbf{P}_{1}\left(\mathbf{B}_{q i}+\Delta \mathbf{B}_{q i}\right)+\mathbf{W}_{1}, \mathbf{P}_{2}\left(\mathbf{B}_{q i}+\Delta \mathbf{B}_{q i}\right)\right. \\
& \left.-\mathbf{P}_{2} \mathbf{K}_{i}\left(\mathbf{D}_{q i}+\Delta \mathbf{D}_{q i}\right)+\mathbf{W}_{2}\right\}, \\
\Upsilon_{13}= & {\left[\mathbf{P}_{2}\left(\mathbf{E}_{q 1 i}+\Delta \mathbf{E}_{q 1 i}\right)-\mathbf{P}_{2} \mathbf{K}_{i}\left(\mathbf{E}_{2 i}+\Delta \mathbf{E}_{2 i}\right)\right], } \\
\Upsilon_{15}= & \operatorname{diag}\left\{\left(\mathbf{A}_{q i}+\Delta \mathbf{A}_{q i}\right)^{T} \mathbf{R}_{1},\left(\mathbf{A}_{q i}+\Delta \mathbf{A}_{q i}\right)^{T} \mathbf{R}_{2}-\left(\mathbf{C}_{q i}+\Delta \mathbf{C}_{q i}\right)^{T} \mathbf{K}_{i}^{T} \mathbf{R}_{2}\right\}, \\
\Upsilon_{25}= & \operatorname{diag}\left\{\left(\mathbf{B}_{q i}+\Delta \mathbf{B}_{q i}\right)^{T} \mathbf{R}_{1}\left(\mathbf{B}_{q i}+\Delta \mathbf{B}_{q i}\right)^{T} \mathbf{R}_{2}-\left(\mathbf{D}_{q i}+\Delta \mathbf{D}_{q i}\right)^{T} \mathbf{K}_{i}^{T} \mathbf{R}_{2}\right\}, \\
\Upsilon_{35}= & {\left[\left(\mathbf{E}_{q 1 i}+\Delta \mathbf{E}_{q 1 i}\right)^{T} \mathbf{R}_{1}\left(\mathbf{E}_{q 1 i}+\Delta \mathbf{E}_{q 1 i}\right)^{T} \mathbf{R}_{2}-\left(\mathbf{E}_{2 i}+\Delta \mathbf{E}_{2 i}\right)^{T} \mathbf{K}_{i}^{T} \mathbf{R}_{2}\right] . }
\end{aligned}
$$

By applying the Schur complement, equation (37) is equivalent to

$$
\sum_{i=1}^{r} h_{i}^{2}(f(t)) \boldsymbol{\Omega}_{i i}+\sum_{i<j}^{r} h_{i}(f(t)) h_{j}(f(t))\left(\boldsymbol{\Omega}_{i j}+\boldsymbol{\Omega}_{j i}\right)<0 .
$$

Thus, the inequalities (30) and (31) hold. The proof is completed.

\section{Optimal Transformation Matrix Selection}

It can readily be obtained that the estimation precision is directly affected by the estimation of the unknown states 
(health parameters). Thus, the key of the parameters estimation is to find an optimal transformation matrix $\mathbf{V}^{*}$, which can ensure that the low-dimensional tuning vector $\mathbf{q}$ can represent as much of the information of the health parameters as possible.

In this paper, the optimization objective is to find an optimal transformation matrix $\mathbf{V}^{*}$, which can ensure that the estimation error of the measured outputs $\rho=(\mathbf{y}-\hat{y})^{T}(\mathbf{y}-\hat{y})$ is minimum.

Thus, the optimization problem can be described as

$$
\begin{array}{ll}
\min & \rho \\
\text { s.t. } & \rho=(\mathbf{y}-\hat{y})^{T}(\mathbf{y}-\hat{y}) .
\end{array}
$$

And the method based on iterative solution is used in this paper, and the process of optimal transformation matrix selection is shown in Figure 1:

(1) To begin with, the initial value of $\mathbf{V}^{*}$ is given randomly. And during the estimation process, the optimal $\mathbf{V}^{*}$ of the last moment is used as the initial value to reduce the quantity of calculation. In order to avoid the calculation converging to a poorly scaled result, the Frobenius norm of $\mathbf{V}^{*}$ must satisfy $\left\|V^{*}\right\|_{F}=1$.

(2) With reference to equation (7), construct the reduced-order state-space model.

(3) Solve the parameters of the $H \infty$ filter.

(4) Calculate estimated error using equation (40).

(5) Determine whether the estimated error $\rho$ achieves convergence within a tolerance (user-specified):

(1) If converged, skip step 6 and proceed directly to step 7.

(2) If not converged, proceed to step 6.

(3) If the number of iterations exceeds 100, skip step 6 and proceed directly to step 7 to meet the realtime requirements.

(6) Use the MATLAB lsqnonlin function to update $\mathbf{V}^{*}$, and the new value still needs to satisfy $\left\|V^{*}\right\|_{F}=1$.

(7) Return the optimal value of $\mathbf{V}^{*}$, and ends.

\section{Simulation Example}

Before proceeding with the simulation, the high-precision aero-thermodynamic mathematical model of aeroengine in full envelope is the basis of the simulation. It can not only build the small deviation dynamic state space models, but can also replace the real engine in simulation. The detailed modeling process is carried out by reference to $[25,26]$. This paper takes a type of double-rotor turbofan engine as research object and uses modeling method based on component characteristics. The afterburner is not considered in this engine model. For greater adaptability, faster calculation speed, and stronger convergence, the self-tuning Broyden quasi-Newton method [27] is used to solve equilibrium equations in the component-

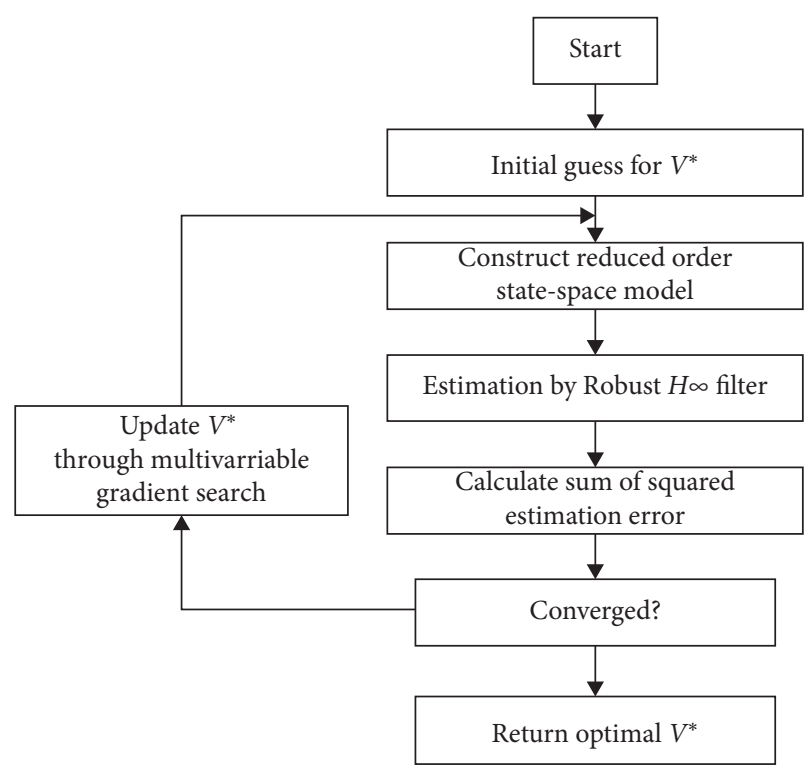

FIgURE 1: Flowchart of $\mathbf{V}^{*}$ iterative search.

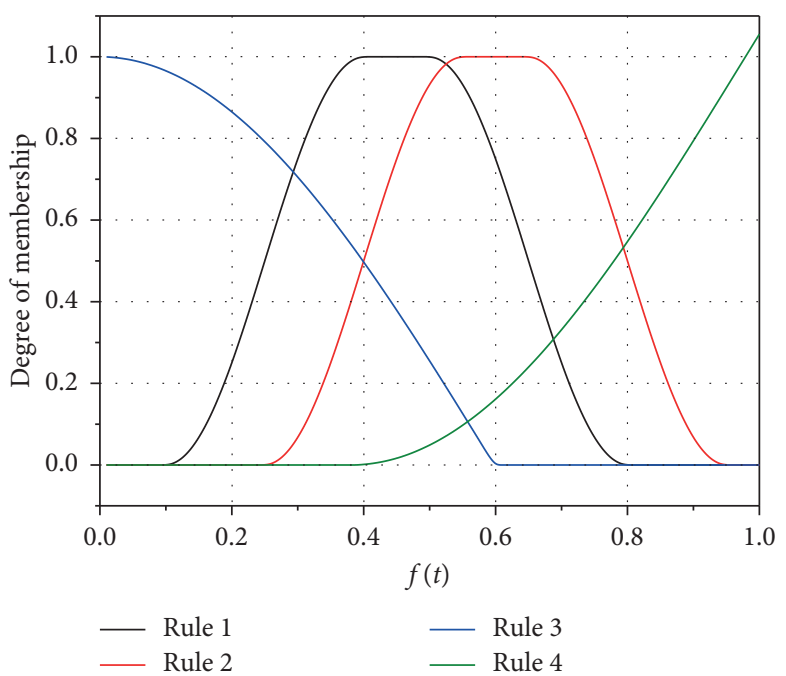

FIgURE 2: Initial membership function.

level mathematical model. And the small deviation dynamic model is solved by fitting method [28-30].

Assuming that the aeroengine works in the maximum state and then based on the methods of flight envelop division in [31-33], a T-S fuzzy model is constructed and the initial membership function is shown in Figure 2. In this paper, the T-S fuzzy model has two state variables, four health parameters, two control inputs, five measured outputs, and three unmeasured outputs, all shown in Table 1.

Then the four T-S fuzzy rules with the selection of $H_{f}$ and Ma can be obtained as follows:

Rule 1. If $H_{f}$ is about $0 \mathrm{~km}$ and $\mathrm{Ma}$ is about 0 , then 
TABLE 1: Variables of aeroengine T-S fuzzy model.

\begin{tabular}{|c|c|c|c|c|}
\hline State variables & Health parameters & Control inputs & Measured outputs & Unmeasured outputs \\
\hline $\begin{array}{l}n_{l} \text {-low-pressure spool } \\
\text { speed }\end{array}$ & $\begin{array}{c}\text { Low-pressure compressor (LPC) } \\
\text { efficiency }\end{array}$ & $w f$-fuel flow & $\begin{array}{l}n_{l} \text {-low-pressure spool } \\
\text { speed }\end{array}$ & $\begin{array}{l}T_{5}-\mathrm{LPT} \text { exit total } \\
\text { temperature }\end{array}$ \\
\hline \multirow{4}{*}{$\begin{array}{l}n_{h} \text {-high-pressure spool } \\
\text { speed }\end{array}$} & LPC flow capacity & \multirow{4}{*}{$\begin{array}{c}A_{8} \text { - nozzle } \\
\text { area }\end{array}$} & $\begin{array}{c}n_{h}-\text { high-pressure spool } \\
\text { speed }\end{array}$ & $F_{n}$-net thrust \\
\hline & Low-pressure turbine (LPT) & & $P_{2 \mathrm{~b}}-\mathrm{LPC}$ exit total & \multirow[b]{3}{*}{$\Pi_{\mathrm{T}}$-turbine pressure ratio } \\
\hline & efficiency & & $\begin{array}{c}\text { pressure } \\
T_{2 b}-\text { LPC exit total }\end{array}$ & \\
\hline & LPT flow capacity & & $\begin{array}{c}T_{3}-\mathrm{HPC} \text { exit total } \\
\text { temperature }\end{array}$ & \\
\hline
\end{tabular}

$$
\begin{aligned}
& \mathbf{A}_{1}=\left[\begin{array}{cc}
-1.26 & 1.02 \\
-0.14 & -1.24
\end{array}\right] \\
& \mathbf{B}_{1}=\left[\begin{array}{ll}
0.35 & 0.54 \\
0.36 & 0.32
\end{array}\right] \text {, } \\
& \mathbf{L}_{1}=\left[\begin{array}{cccc}
-1.02 & 0.90 & -0.62 & 0.51 \\
-0.26 & -0.11 & 0.28 & 0.23
\end{array}\right] \text {, } \\
& \mathbf{E}_{11}=\left[\begin{array}{c}
-0.5 \\
1.0
\end{array}\right] \text {, } \\
& \mathrm{C}_{1}=\left[\begin{array}{cc}
1 & 0 \\
0 & 1 \\
0.55 & -0.29 \\
0.51 & -0.07 \\
0.36 & 0.54
\end{array}\right] \text {, } \\
& \mathbf{D}_{1}=\left[\begin{array}{cc}
0 & 0 \\
0 & 0 \\
0.19 & -0.82 \\
0.05 & -0.22 \\
0.04 & -0.16
\end{array}\right] \text {, } \\
& \mathbf{M}_{1}=\left[\begin{array}{cccc}
0 & 0 & 0 & 0 \\
0 & 0 & 0 & 0 \\
0.94 & -0.18 & 0.02 & -0.07 \\
0.24 & -0.39 & 0.01 & -0.02 \\
0.13 & -0.23 & -0.09 & -0.08
\end{array}\right] \text {, } \\
& \mathbf{E}_{21}=\left[\begin{array}{c}
0 \\
0 \\
0.2 \\
0.1 \\
0.15
\end{array}\right] \\
& \mathbf{F}_{1}=\left[\begin{array}{cc}
0.25 & -0.54 \\
-0.14 & 1.27 \\
0.40 & 0.04
\end{array}\right] \text {, } \\
& \mathbf{G}_{1}=\left[\begin{array}{cc}
0.45 & 0.09 \\
-0.10 & 0.39 \\
0.24 & 0.40
\end{array}\right] \text {, } \\
& \mathbf{N}_{1}=\left[\begin{array}{cccc}
-0.23 & -0.29 & 0.07 & -0.18 \\
-0.09 & 0.26 & -0.33 & -0.19 \\
0.81 & -0.08 & 0.04 & -0.08
\end{array}\right] \text {. }
\end{aligned}
$$

Rule 2. If $H_{f}$ is about $4.8 \mathrm{~km}$ and $\mathrm{Ma}$ is about 1.24 , then

$$
\begin{aligned}
& \mathbf{A}_{2}=\left[\begin{array}{cc}
-2.66 & 1.10 \\
-0.61 & -1.39
\end{array}\right] \text {, } \\
& \mathbf{B}_{2}=\left[\begin{array}{ll}
0.39 & 0.42 \\
0.36 & 0.33
\end{array}\right] \text {, } \\
& \mathbf{L}_{2}=\left[\begin{array}{cccc}
-0.86 & 0.87 & -0.64 & 0.52 \\
-0.28 & -0.10 & 0.27 & 0.23
\end{array}\right] \text {, } \\
& \mathbf{E}_{12}=\left[\begin{array}{c}
-0.3 \\
0.1
\end{array}\right] \text {, } \\
& \mathbf{C}_{2}=\left[\begin{array}{cc}
1 & 0 \\
0 & 1 \\
2.03 & -0.34 \\
0.66 & -0.06 \\
0.42 & 0.58
\end{array}\right] \\
& \mathbf{D}_{2}=\left[\begin{array}{cc}
0 & 0 \\
0 & 0 \\
0.14 & -0.67 \\
0.02 & -0.11 \\
0.02 & -0.10
\end{array}\right] \text {, } \\
& \mathbf{M}_{2}=\left[\begin{array}{cccc}
0 & 0 & 0 & 0 \\
0 & 0 & 0 & 0 \\
0.92 & -0.16 & 0.02 & -0.05 \\
0.15 & -0.30 & 0 & -0.01 \\
0.08 & -0.18 & -0.09 & -0.07
\end{array}\right] \\
& \mathbf{E}_{22}=\left[\begin{array}{c}
0 \\
0 \\
0.3 \\
0.25 \\
0.1
\end{array}\right] \text {, } \\
& \mathbf{F}_{2}=\left[\begin{array}{cc}
-0.34 & -0.52 \\
-0.06 & 1.26 \\
2.10 & 0.12
\end{array}\right] \text {, } \\
& \mathbf{G}_{2}=\left[\begin{array}{cc}
0.45 & 0.09 \\
-0.10 & 0.43 \\
0.54 & -0.57
\end{array}\right] \text {, } \\
& \mathbf{N}_{2}=\left[\begin{array}{cccc}
-0.31 & -0.22 & 0.08 & -0.17 \\
0 & 0.19 & -0.34 & -0.19 \\
0.94 & -0.18 & 0.09 & -0.19
\end{array}\right]
\end{aligned}
$$


Rule 3. If $H_{f}$ is about $12.8 \mathrm{~km}$ and $\mathrm{Ma}$ is about 0.7 , then

$$
\begin{aligned}
& \mathbf{A}_{3}=\left[\begin{array}{cc}
-0.31 & 0.19 \\
-0.05 & -0.28
\end{array}\right] \text {, } \\
& \mathbf{B}_{3}=\left[\begin{array}{ll}
0.11 & 0.11 \\
0.09 & 0.09
\end{array}\right] \text {, } \\
& \mathbf{L}_{3}=\left[\begin{array}{cccc}
-0.24 & 0.21 & -0.16 & 0.13 \\
-0.07 & -0.02 & 0.07 & 0.06
\end{array}\right] \text {, } \\
& \mathbf{E}_{13}=\left[\begin{array}{l}
0.2 \\
0.3
\end{array}\right] \text {, } \\
& \mathbf{C}_{3}=\left[\begin{array}{cc}
1 & 0 \\
0 & 1 \\
0.53 & -0.18 \\
0.49 & -0.04 \\
0.35 & 0.55
\end{array}\right] \text {, } \\
& \mathbf{D}_{3}=\left[\begin{array}{cc}
0 & 0 \\
0 & 0 \\
0.19 & -0.84 \\
0.05 & -0.21 \\
0.04 & -0.15
\end{array}\right] \text {, } \\
& \mathbf{M}_{3}=\left[\begin{array}{cccc}
0 & 0 & 0 & 0 \\
0 & 0 & 0 & 0 \\
0.94 & -0.17 & 0.02 & -0.08 \\
0.23 & -0.41 & 0.01 & -0.02 \\
0.13 & -0.25 & -0.10 & -0.09
\end{array}\right] \text {, } \\
& \mathbf{E}_{23}=\left[\begin{array}{c}
0 \\
0 \\
0.1 \\
0.2 \\
0.1
\end{array}\right] \text {, } \\
& \mathbf{F}_{3}=\left[\begin{array}{cc}
0.22 & -0.31 \\
-0.10 & 1.04 \\
0.59 & 0.12
\end{array}\right] \text {, } \\
& \mathbf{G}_{3}=\left[\begin{array}{cc}
0.45 & 0.12 \\
-0.08 & 0.36 \\
0.45 & -0.58
\end{array}\right] \text {, } \\
& \mathbf{N}_{3}=\left[\begin{array}{cccc}
-0.25 & -0.25 & 0.07 & -0.20 \\
-0.07 & 0.21 & -0.34 & -0.19 \\
1.05 & -0.15 & 0.07 & -0.17
\end{array}\right] \text {. }
\end{aligned}
$$

Rule 4. If $H_{f}$ is about $18.8 \mathrm{~km}$ and $\mathrm{Ma}$ is about 1.72 , then

$$
\begin{aligned}
& \mathbf{A}_{4}=\left[\begin{array}{cc}
-0.63 & 0.24 \\
-0.13 & -0.29
\end{array}\right] \text {, } \\
& \mathbf{B}_{4}=\left[\begin{array}{ll}
0.08 & 0.10 \\
0.08 & 0.07
\end{array}\right] \text {, } \\
& \mathbf{L}_{4}=\left[\begin{array}{cccc}
-0.20 & 0.19 & -0.14 & 0.11 \\
-0.06 & -0.02 & 0.06 & 0.05
\end{array}\right], \\
& \mathbf{E}_{14}=\left[\begin{array}{l}
0.3 \\
0.5
\end{array}\right] \text {, } \\
& \mathbf{C}_{4}=\left[\begin{array}{cc}
1 & 0 \\
0 & 1 \\
2.29 & -0.36 \\
0.73 & -0.07 \\
0.46 & 0.58
\end{array}\right] \\
& \mathbf{D}_{4}=\left[\begin{array}{cc}
0 & 0 \\
0 & 0 \\
0.14 & -0.66 \\
0.03 & -0.12 \\
0.03 & -0.11
\end{array}\right] \text {, } \\
& \mathbf{M}_{4}=\left[\begin{array}{cccc}
0 & 0 & 0 & 0 \\
0 & 0 & 0 & 0 \\
0.95 & -0.16 & 0.02 & -0.05 \\
0.18 & -0.30 & 0 & -0.01 \\
0.09 & -0.17 & -0.09 & -0.08
\end{array}\right] \text {, } \\
& \mathbf{E}_{24}=\left[\begin{array}{c}
0 \\
0 \\
0.2 \\
0.1 \\
0.1
\end{array}\right] \text {, } \\
& \mathbf{F}_{4}=\left[\begin{array}{cc}
-0.37 & -0.50 \\
-0.07 & 1.25 \\
2.00 & 0.14
\end{array}\right] \text {, } \\
& \mathbf{G}_{4}=\left[\begin{array}{cc}
0.46 & 0.05 \\
-0.11 & 0.47 \\
0.61 & -0.43
\end{array}\right] \text {, } \\
& \mathbf{N}_{4}=\left[\begin{array}{cccc}
-0.28 & -0.21 & 0.07 & -0.18 \\
-0.02 & 0.18 & -0.33 & -0.18 \\
0.79 & -0.20 & 0.09 & -0.22
\end{array}\right] \text {. }
\end{aligned}
$$



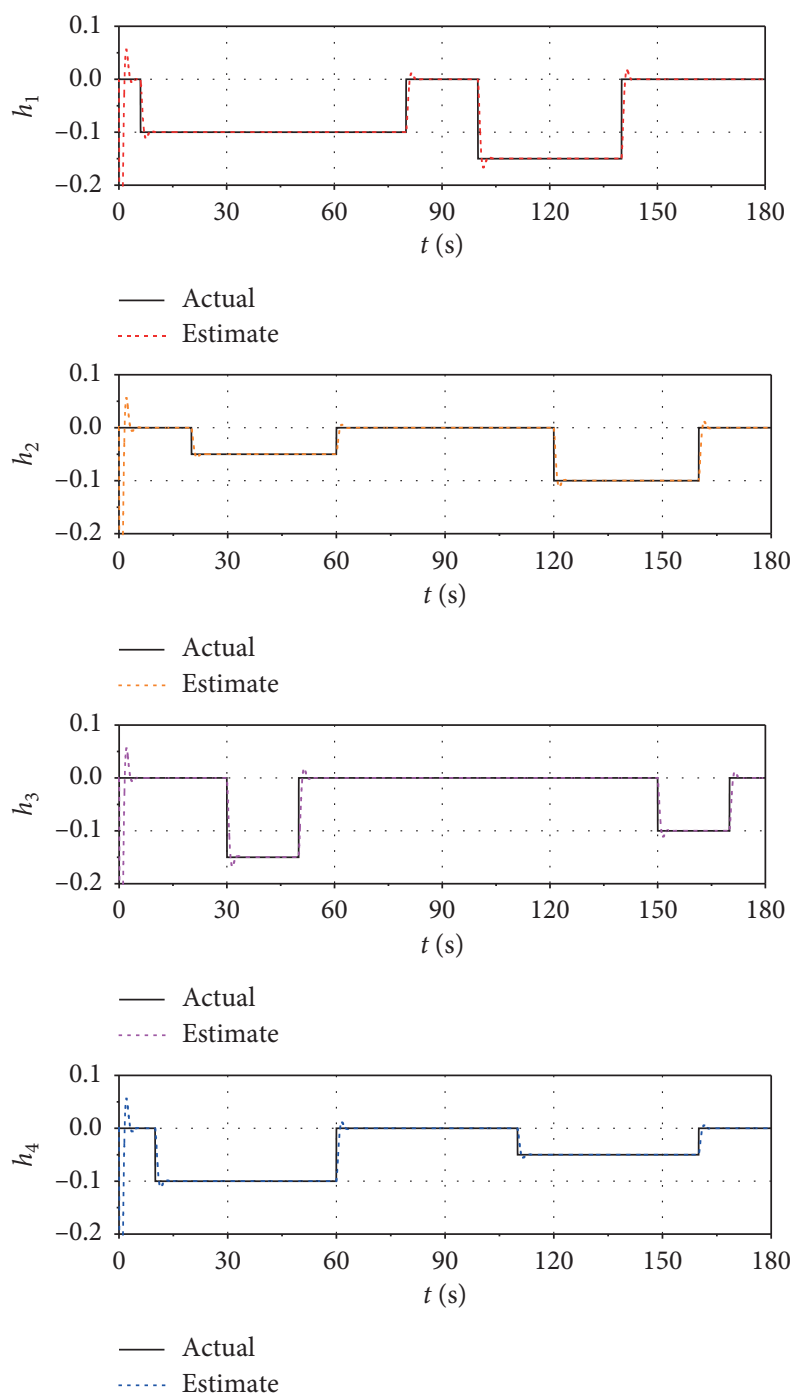

Figure 3: The estimation of health parameters for Type I.

Moreover, the uncertainties of the system are

$$
\begin{aligned}
& \mathbf{U}_{1 i}=\left[\begin{array}{lllll}
0.2 & -0.3 & 0 & 0 & 0
\end{array}\right]^{T}, \\
& \mathbf{V}_{4 i}=\left[\begin{array}{lll}
-0.1 & 0.2 & 0.15
\end{array}\right], \\
& \mathbf{U}_{2 i}=\left[\begin{array}{lllll}
0.1 & 0.3 & -0.1 & 0.2 & 0.05
\end{array}\right]^{T}, \\
& \mathbf{V}_{3 i}=0.1 \text {, } \\
& \mathbf{V}_{1 i}=\left[\begin{array}{lllll}
0.1 & 0.5 & 0 & 0 & 0
\end{array}\right], \\
& \mathbf{V}_{2 i}=\left[\begin{array}{lllll}
0.2 & -0.1 & 0 & 0 & 0
\end{array}\right] \text {, } \\
& (i=1,2,3,4) \text {. }
\end{aligned}
$$

The system sampling period is set as $T=20 \mathrm{~ms}$, and the external disturbance $w(t)$ is

$$
w(t)=\frac{0.3 \sin (0.8 t)}{5 \sin (6 t)+3 \cos (8 t)} .
$$

The state space of time-delay is $\tau(t) \in\{1,2,3\}$, and the state transition matrix of $\tau(t)$ is
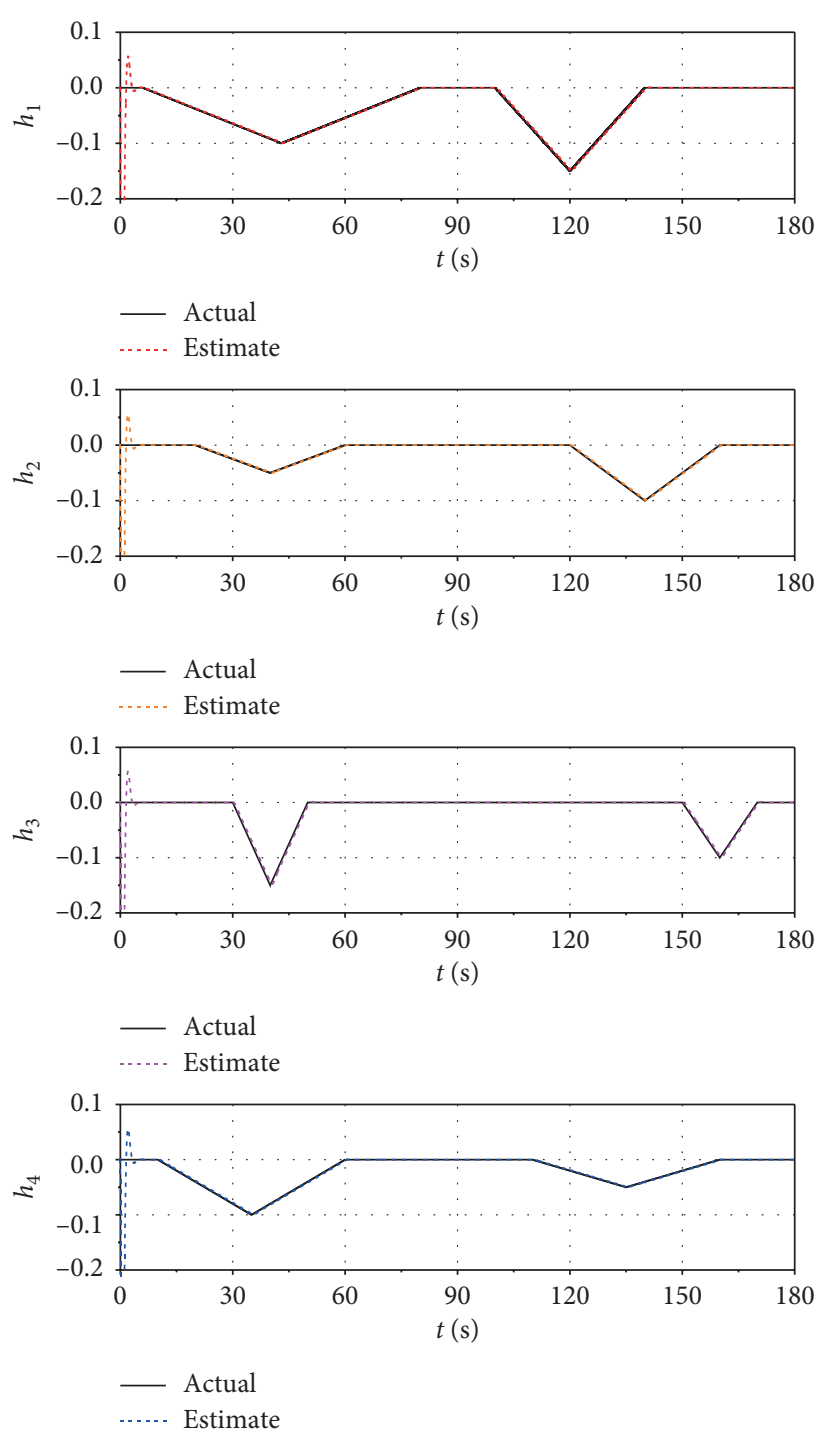

FIgURE 4: The estimation of health parameters for Type II.

$$
\Pi=\left[\begin{array}{ccc}
-0.6 & 0.4 & 0.2 \\
0.3 & -0.5 & 0.2 \\
0.1 & 0.4 & -0.5
\end{array}\right] \text {. }
$$

The working condition is chosen as $H=5 \mathrm{~km}$ and $\mathrm{Ma}=0.3$. The given value of $\gamma$ is $\gamma=2$.

Two types of performance degradation are selected to simulate, which are slow varying and sudden change of health parameters. For the sudden change type (Type I), there is a step change for health parameters, and this type is used to represent the sudden damage, such as foreign objects damage. And the slow varying type (Type II) is defined as the linear variation of health parameters to represent the gradual performance degradation during using process. These two types can cover almost all the health parameters changes in practice, and they are simulated respectively to illustrate the effectiveness of the method proposed in this paper.

The results are shown in Figures 3-6. It can be seen that the method proposed in this paper can effectively simulate 

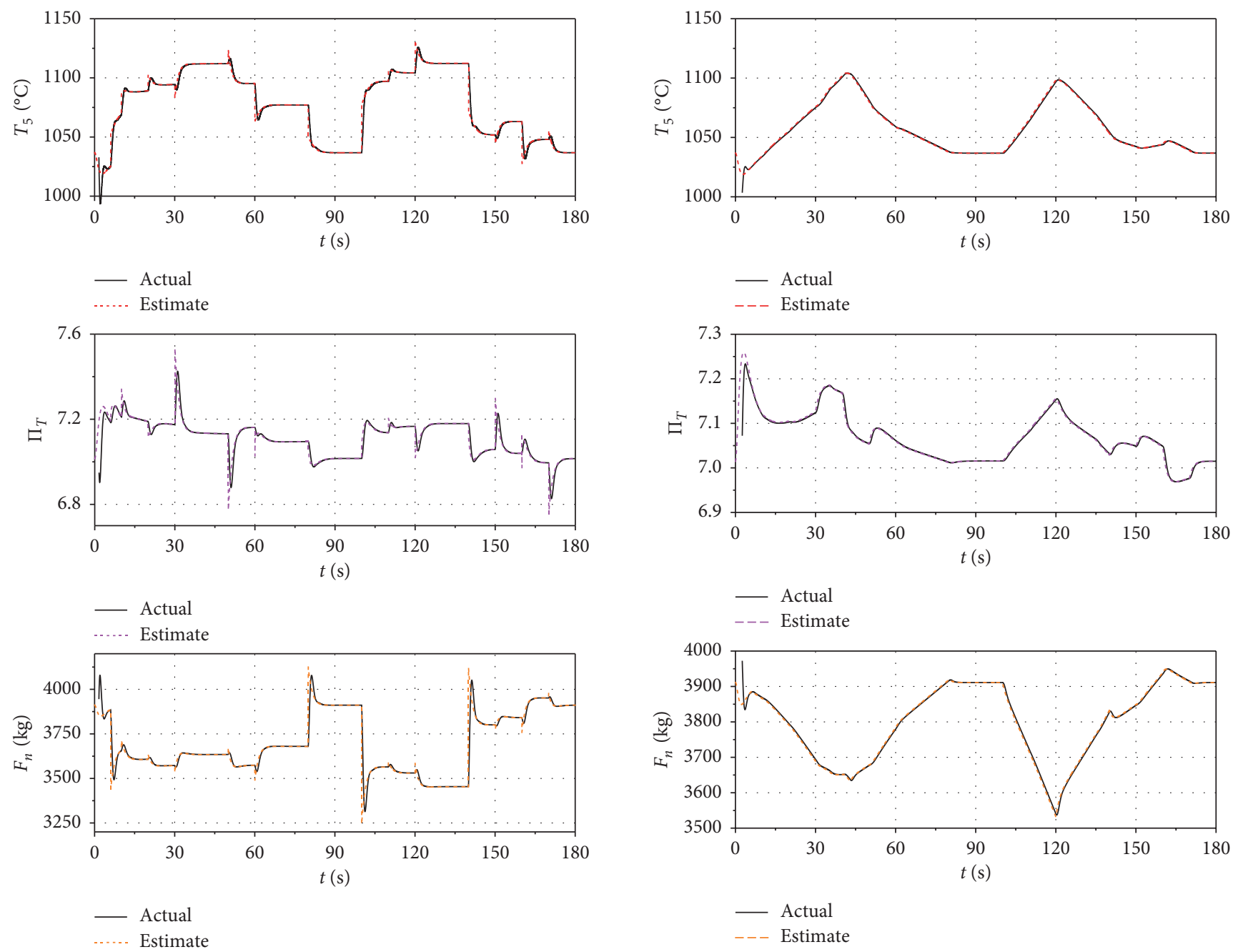

Figure 5: The estimation of performance parameter for Type I.

the health and performance parameters of the aeroengine. The estimated values can accurately track the changes as the health parameters shift.

By comparing the results of the two types of health parameters change, it can be concluded that the estimated values for slow varying type are more accurate. For the type of sudden change, it takes a while for the estimated values to stabilize and the overshoot is slightly larger.

Table 2 shows the average estimated error of the health and performance parameters. It can be seen that the estimated error is within $2.5 \%$ for all types. At the same time, the results are more accurate except for the beginning of simulation.

Furthermore, the results show that the method proposed in this paper can accurately estimate most system parameters of interest. On this basis, the method can also be used in the investigations of direct control, fault diagnosis, health management, and online monitoring. And for aeroengine, there are already some researches which indicate that the changes of health parameters can characterize specific faults, allowing for online monitoring and alerting [34-36].

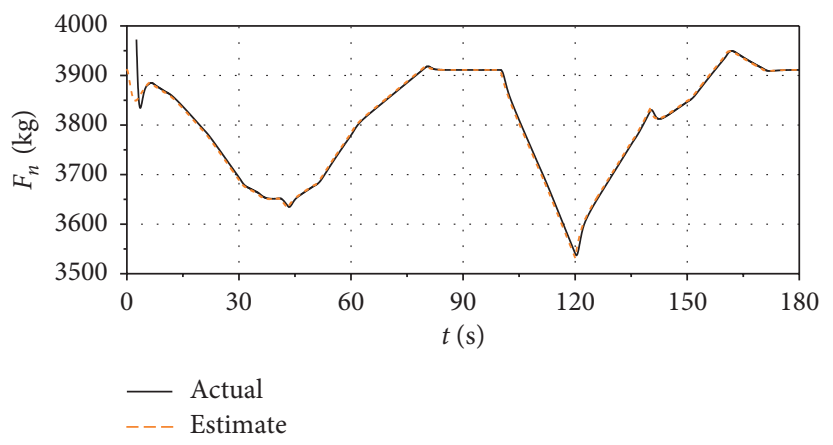

FIgURE 6: The estimation of performance parameter for Type II.

TABLE 2: Estimated errors of health and performance parameters.

\begin{tabular}{lcc}
\hline Estimated error & Type I (\%) & Type II (\%) \\
\hline$h_{1}$ & 0.46 & 0.40 \\
$h_{2}$ & 0.38 & 0.31 \\
$T_{5}$ & 1.27 & 0.98 \\
$F_{n}$ & 1.95 & 1.57 \\
$\Pi_{\mathrm{T}}$ & 2.46 & 2.03 \\
\hline
\end{tabular}

\section{Conclusion}

In this paper, a method is proposed for the underdetermined estimation problem of the distributed control systems. First, the T-S fuzzy model for DCSs is constructed and a model tuning parameter vector of appropriate dimension is produced. Then an observer-based fuzzy filter is designed and the sufficient condition for the existence of the designed filter is derived in terms of LMIs solutions. Besides, the method based on iterative solution is used to select the optimal transformation matrix to minimize the estimated error. Finally, the results of the simulation show that the proposed method can effectively estimate unmeasured parameters of the aeroengine and the estimated error is less than $2.5 \%$. 


\section{Data Availability}

The data used to support the findings of this study are included within the article.

\section{Conflicts of Interest}

The authors declare that they have no conflicts of interest.

\section{Acknowledgments}

This work was supported by the National Natural Science Foundation of China (51476187, 51606219, and 51506221).

\section{References}

[1] M. Khalgui and O. Mosbahi, "Intelligent distributed control systems," Information and Software Technology, vol. 52, no. 12, pp. 1259-1271, 2010.

[2] A. Behbahani and B. Tulpule, "Perspective for distributed intelligent engine controls of the future," in Proceeding of the 46th AIAA/ASME/SAE/ASEE Joint Propulsion Conference \& Exhibit, Nashville, TN, USA, July 2010.

[3] J. P. Hespanha, P. Naghshtabrizi, and Y. Xu, "A survey of recent results in networked control systems," Proceedings of the IEEE, vol. 95, no. 1, pp. 138-162, 2007.

[4] G. C. Goodwin, E. I. Silva, and D. E. Quevedo, "A brief introduction to the analysis and design of networked control systems," Automatica, vol. 18, pp. 810-820, 2008.

[5] F. Kazempour and J. Ghaisari, "Stability analysis of modelbased networked distributed control systems," Journal of Process Control, vol. 23, no. 3, pp. 444-452, 2013.

[6] Y. Kao, J. Xie, C. Wang, and H. R. Karimi, "A sliding mode approach to Ho non-fragile observer-based control design for uncertain Markovian neutral-type stochastic systems," Automatica, vol. 52, pp. 218-226, 2015.

[7] D. L. Simon and A. W. Rinehart, "Sensor selection for aircraft engine performance estimation and gas path fault diagnostics," Journal of Engineering for Gas Turbines and Power, vol. 138, no. 7, pp. 1-11, 2016.

[8] J. T. Csank and J. W. Connolly, "Model-based engine control architecture with an extended kalman filter," in Proceedings of the AIAA Guidance, Navigation and Control Conference, San Diego, CA, USA, January 2016.

[9] G. Liao, Y. Jiao, Q. Li, and J. Huang, "Research on high accuracy real-time component-level modeling method for turbo-shaft engine," Journal of Propulsion Technology, vol. 37, no. 1, pp. 25-33, 2016.

[10] D. L. Simon, J. B. Armstrong, and S. Garg, "Application of an optimal tuner selection approach for on-board self-tuning engine models," Journal of Engineering for Gas Turbine and Power, vol. 134, no. 4, pp. 1-11, 2012.

[11] Z. Zhang, C. Lin, and B. Chen, "New decentralized Ho filter design for nonlinear interconnected systems based on TakagiSugeno fuzzy models," IEEE Transactions on Cybernetics, vol. 45, no. 12, pp. 2914-2924, 2015.

[12] G. H. Yang and X. G. Guo, "Brief paper: insensitive $H \infty$ filter design for continuous-time systems with respect to filter coefficient variations," Automatica, vol. 46, pp. 1860-1869, 2010.

[13] Y. Chen, Z. Wang, and Y. Yuan, "Distributed Ho filtering for switched stochastic delayed systems over sensor networks with fading measurements," IEEE Transactions on Cybernetics, vol. 50, no. 1, pp. 2-14, 2020.
[14] T. Brotherton, A. Volponi, R. Luppold, and D. L. Simon, "eSTORM: enhanced self turning on-board real-time engine model," in Proceedings of the 2003 IEEE Aerospace Conference, Big Sky, MT, USA, March 2003.

[15] T. Kobayashi and D. L. Simon, "Application of a bank of Kalman filters for aircraft engine fault diagnostics," in Proceedings of the ASME Turbo Exposition, Atlanta, GA, USA, June 2003.

[16] T. Kobayashi and D. L. Simon, "Evaluation of an enhanced bank of Kalman filters for in-flight aircraft engine sensor fault diagnostics," in Proceedings of the ASME Turbo Exposition, Vienna, Austria, June 2004.

[17] J. S. Litt, "An optimal orthogonal decomposition method for kalman filter-based turbofan engine thrust estimation," Journal of Engineering for Gas Turbine and Power, vol. 130, pp. 1-12, 2008.

[18] R. C. Magana and A. Medina, "Time domain transient state estimation using singular value decomposition Poincare map and extrapolation to the limit cycle," International Journal of Electrical Power\&Energy Systems, vol. 53, pp. 810-817, 2013.

[19] D. L. Simon and S. Garg, "Optimal tuner selection for kalman filter based aircraft engine performance estimation," Journal of Engineering for Gas Turbine and Power, vol. 132, pp. 1-10, 2010.

[20] Y. Yu, H. K. Lam, and K. Y. Chan, “T-S fuzzy model based output feedback tracking control with control input saturation," IEEE Transactions on Fuzzy Systems, vol. 26, no. 6, pp. 3514-3523, 2018.

[21] N. Rong, Z. Wang, and H. Zhang, "Finite-time stabilization for discontinuous interconnected delayed systems via interval type-2 T-S fuzzy model approach," IEEE Transactions on Fuzzy Systems, vol. 27, no. 2, pp. 249-261, 2019.

[22] C. Peng, S. Ma, and X. Xie, "Observer-based non-PDC control for networked T-S fuzzy systems with an event-triggered communication," IEEE Transactions on Cybernetics, vol. 47, no. 8, pp. 2279-2287, 2017.

[23] N. Vatanski, J. Georges, and C. Aubrun, "Networked control with delay measurement and estimation," Control Engineering Practice, vol. 17, no. 2, pp. 231-244, 2008.

[24] T. Iwasaki and R. E. Skelton, "All controllers for the general $H \infty$ control problem: LMI existence condition and state space formulas," Automatica, vol. 30, pp. 1307-1317, 1994.

[25] G. Q. Luo, Numerical Methods for Aviation Gas Turbine Engine Simulation, National Defence Industry Press, Beijing, China, 2007.

[26] R. John, "Real-time simulation of F100-PW-100 turbofan engine using the hybrid computer," Computer-Aided Design, vol. 8, no. 2, pp. 1-8, 1976.

[27] Y. Wang, Q. Li, and X. Huang, "Numerical calculation of aero-engine model based on self-tuning Broyden quasiNewton method," Journal of Aerospace Power, vol. 31, no. 1, pp. 249-256, 2016.

[28] Q. Li and J. Sun, "Aero-engine variable modeling based on the genetic algorithm," Journal of Aerospace Power, vol. 24, no. 2, pp. 427-431, 2006.

[29] T. Zheng and X. Wang, "Modified method of establishing the state space model of aero-engine," Journal of Propulsion Technology, vol. 26, no. 1, pp. 46-49, 2005.

[30] N. Sugiyama, "Deviation of ABCD system matrices from nonlinear dynamic simulation of jet engines," in Proceedings of the AIAA Conference, Nashville, TN, USA, July 1992.

[31] L. Wang, S. Xie, and Z. Miao, "Identification of T-S fuzzy model for aero-engine based on flight envelop division," 
Journal of Aerospace Power, vol. 28, no. 5, pp. 1159-1165, 2013.

[32] X. Zhai, S. Xie, and Z. Miao, "Fault detection of aero-engine non-linear distributed control system based on T-S fuzzy model," Journal of Aerospace Power, vol. 28, no. 6, pp. 1429-1435, 2013.

[33] L. Wang, S. Xie, and L. Ren, "Fault diagnosis of sensors in distributed control system based on T-S fuzzy KPCA model," Journal of Propulsion Technology, vol. 35, no. 7, pp. 988-995, 2014.

[34] A. Stamatis, K. Mathioudakis, and K. D. Papailiou, "Adaptive simulation of gas turbine performance," Journal of Engine Gas Turbines Power, vol. 112, pp. 168-175, 1990.

[35] A. Tsalavoutas, K. Mathioudakis, and A. Stamatis, "Identifying faults in the variable geometry system of a gas turbine compresso," Journal of Turbomachinery, vol. 123, pp. 33-39, 2001.

[36] S. Ogaji, S. Sampat, and R. Singh, "Parameter selection for diagnosing a gas-turbine's performance deterioration," Applied Energy, vol. 73, no. 1, pp. 25-46, 2002. 